\title{
Exogenous hydrogen sulfide mitigates the fatty liver in obese mice through improving lipid metabolism and antioxidant potential
}

Dongdong $\mathrm{Wu}^{1 \dagger}$, Nairui Zheng ${ }^{2,3 \dagger}$, Kunqing $\mathrm{Qi}^{4 \dagger}$, Huijun Cheng ${ }^{1}$, Ziqiang Sun ${ }^{1}$, Biao Gao ${ }^{1}$, Youjing Zhang ${ }^{1}$, Wuyan Pang ${ }^{5}$, Chaoshen Huangfu ${ }^{1,2}$, Shaoping $\mathrm{Ji}^{1}$, Mengzhou Xue ${ }^{4^{*}}$, Ailing $\mathrm{Ji}^{\mathrm{i}^{*}}$ and Yanzhang $\mathrm{Li}^{\mathrm{i}^{*}}$

\begin{abstract}
Background: Nonalcoholic fatty liver disease (NAFLD) is the most common liver disease in the world. Hydrogen sulfide $(\mathrm{H} 2 \mathrm{~S})$ plays an important role in physiology and pathophysiology of liver. However, whether exogenous $\mathrm{H} 2 \mathrm{~S}$ could mitigate the hepatic steatosis in mice remains unclear. The aim of this study is to evaluate the effects of $\mathrm{H} 2 \mathrm{~S}$ on fatty liver.

Methods: C57BL/6 mice were fed with either a high-fat diet (HFD) or a normal fat diet (NFD) for 16 weeks. After 12 weeks of feeding, the HFD-fed mice were injected one time per day with NaHS or saline for the followed 4 weeks.

Results: Compared to NFD, HFD could induce an accumulation of lipids in liver and a damage of hepatic structure. Compared to saline treatment, in the liver of HFD fed mice H2S treatment could significantly (1) recover the structure; (2) decrease the accumulation of lipids including triglyceride (TG) and total cholesterol (TC); (3) decrease the expression of fatty acid synthase (FAS) and increase the expression of carnitine palmitoyltransferase-1 (CPT-1); (4) reduce malondialdehyde (MDA) levels; (5) increase the activities of superoxide dismutase (SOD) and glutathione peroxidase (GPX).
\end{abstract}

Conclusion: $\mathrm{H} 2 \mathrm{~S}$ could mitigate the fatty liver by improving lipid metabolism and antioxidant potential in HFD-induced obese mice.

Keywords: Hydrogen sulfide, Fatty liver, Mitigation, Lipid metabolism, Antioxidant

\section{Introduction}

Nonalcoholic fatty liver disease (NAFLD) is the most common liver disease in the world, which is caused by abnormal accumulation of triglyceride inside the hepatocytes [1]. NAFLD may progress to non-alcoholic steatohepatitis (NASH), fibrosis, cirrhosis, and hepatocellular cancer, and is also a risk factor for the development of type 2 diabetes and cardiovascular disease (CVD) [2].

Hydrogen sulfide (H2S) is an endogenous signaling molecule in mammalians [3], which is synthesized enzymatically

\footnotetext{
* Correspondence: xuemengzhou@henu.edu.cn; ailingji@163.com; yanzhang206@163.com

${ }^{\dagger}$ Equal contributors

${ }^{4}$ Department of Neurology, the First Affiliated Hospital, Institute of Neurological Disorder, Henan University, Kaifeng 475004, China

${ }^{1}$ Medical College of Henan University, Kaifeng 475004, Henan, China

Full list of author information is available at the end of the article
}

by cystathionine $\beta$-synthase (CBS), cystathionine $\gamma$-lyase (CSE), and 3-mercaptopyruvate sulfurtransferase (MST) during cysteine metabolism [3]. Accumulating evidence suggests that $\mathrm{H} 2 \mathrm{~S}$ plays an important role in physiology and pathophysiology of liver [4]. The dysregulation of endogenous $\mathrm{H} 2 \mathrm{~S}$ is correlated with the symptoms of diabetes [5,6] and liver cirrhosis [7]. Blood levels of H2S in type 2 diabetic patients (T2D) are lower than that of the controls [8]. Endogenous formation of $\mathrm{H} 2 \mathrm{~S}$ is impaired in rats with $\mathrm{NASH}$ and treatment with $\mathrm{H} 2 \mathrm{~S}$ can prevent NASH in rats possibly through abating oxidative stress and suppressing inflammation [9]. The administration of sodium hydrosulfide (NaHS)-a H2S donor in rodents protects against liver injury [4], which is induced by ischemia reperfusion [10], acetaminophen [11], or carbon tetrachloride [7]. Application of H2S also exhibits the effects 
on mitochondrial function [12,13], anti-oxidative stress $[14,15]$, apoptosis [16], inflammation [17], angiogenesis [18-20], and blood pressure [21-23].

Recent studies have demonstrated that CSE, CBS, and MST proteins could be detected in the liver, and they contribute to liver production of $\mathrm{H} 2 \mathrm{~S}$ to different extents [4,9,24-26]. High fat diet can induce a down-regulation of CSE/CBS and results in less $\mathrm{H} 2 \mathrm{~S}$ production in the liver of rodents [25]. Based on this point, we propose that increasing $\mathrm{H} 2 \mathrm{~S}$ by administration of NaHS may restore liver from steatosis in HFD-induced obese mice. To prove our hypotheses, we employed a diet-induced obesity (DIO) mouse model to evaluate the effects of exogenous $\mathrm{H} 2 \mathrm{~S}$ on fatty liver of obese mice. We found that the administration of H2S donor NaHS could mitigate the fatty liver by improving lipid metabolism and antioxidant potential in HFD-induced obese mice. H2S products may be used as therapeutical drugs for the NAFLD.

\section{Materials and methods Animals}

The protocols for animal experiments were reviewed and approved by the Animal Care and Use Committee of Henan University. Eight weeks old male C57BL/6 mice were purchased from Nanjing Biomedical Research Institute of Nanjing University (Nanjing, China) and housed in a temperature and humidity controlled environment on a 12 hour light/dark cycle with free access to food and water. The mice were fed either a chow (normal fat diet, NFD; KeAoXieli, Beijing, China) or a high fat diet (HFD, 45\% kcal as fat, MD12032, Medicience Ltd., Jiangsu, China) for total 16 weeks. After 12 weeks of feeding, the HFD feeding mice were divided into HFD group and HFD $+\mathrm{H} 2 \mathrm{~S}$ group. Each group contained 10 HFD feeding mice. The mice from HFD group and HFD + $\mathrm{H} 2 \mathrm{~S}$ group were received an intraperitoneal (i.p.) injection of either saline $(10 \mathrm{ml} / \mathrm{kg} /$ day) or NaHS (50 umole/kg/day, dissolved in saline, $10 \mathrm{ml} / \mathrm{kg}$ body weight) for 4 weeks. At the end of experiments, the mice were killed. Tissues were rapidly removed from the mice and frozen either in liquid nitrogen or embedded in frozen section compound (Surgipath FSC22, Leica) and stored at $-20^{\circ} \mathrm{C}$ or by immersion in $4 \%$ formalin.

\section{Histological analysis}

For histology, livers were fixed in formalin, embedded in paraffin, and cut into $5 \mu \mathrm{m}$ thick sections. The sections of livers were stained with hematoxylin and eosin. For oil red $\mathrm{O}(\mathrm{ORO})$ staining, the frozen liver tissues were cut into $10 \mu \mathrm{m}$-thick sections, stained with oil red $\mathrm{O}$, and counterstained with hematoxylin [27]. All sections were scanned at an absolute magnification of 200x under a light microscope (Olympus, BX51 microscope, Tokyo, Japan) and analyzed using Image-J software (NIH). The accumulation of hepatic lipid droplets was presented as the percentage of the blank area (lipid droplets, $H \& E$ staining) or the red staining area (lipid droplets, ORO staining) relative to the whole area of the photomicrograph.

\section{Determination of liver triglyceride (TG) and total cholesterol (TC)}

Liver tissues were homogenized on ice with the sample buffer [tissue weight: buffer $=1(\mathrm{~g})$ : $9(\mathrm{ml})]$. The homogenates were centrifuged at $2500 \mathrm{rpm}$ for $10 \mathrm{~min}$. The supernatants were transferred to the new tubes. Triglyceride (TG) and total cholesterol (TC) in the supernatants were measured by enzymatic colorimetric methods using commercial kits according to the manufacturer's protocols (Nanjing Jiancheng Bioengineering Institute, Nanjing, China).

\section{RNA extraction and real-time PCR}

Total RNA was isolated from liver tissues using TRIzol reagent (life technologies), treated with DNase I (Roch), and purified using an RNA Clean Up Kit (Kangwei, China). One microgram of total RNA was used for cDNA synthesis using a cDNA Reverse Transcription Kit (Kangwei, China). The primer sequences for fatty acid synthase (FAS) [28], carnitine palmitoyltransferase-1 (CPT-1) [29], and 18s rRNA [30] are shown in Table 1. PCR tests were performed in a total volume of $20 \mu \mathrm{l}$ using the following thermal cycling parameters: $94^{\circ} \mathrm{C}$ for $2 \mathrm{~min}$, and then 40 cycles of $94^{\circ} \mathrm{C}$ for $30 \mathrm{~s}, 58^{\circ} \mathrm{C}$ for $30 \mathrm{~s}$, and $72^{\circ} \mathrm{C}$ for $1 \mathrm{~min}$. The mRNA expression levels of the test genes were normalized to 18s rRNA levels.

\section{Measurement of hepatic FAS and CPT-1 levels}

A small piece of the frozen liver tissue was homogenized in PBS (pH 7.2-7.4) on ice by manual grinding. Insoluble material was removed by centrifugation for $15 \mathrm{~min}$ at $2000 \mathrm{rpm}, 4^{\circ} \mathrm{C}$. Hepatic FAS and CPT-1 levels were measured using ELISA kits according to the manufacturer's protocol (Tianchi, Zhengzhou, China).

Table 1 Sequences of primers used in the study

\begin{tabular}{|c|c|c|c|}
\hline Genes & Forward (5'-3') & Reverse $\left(5^{\prime}-3^{\prime}\right)$ & GenBank accession \\
\hline 18s rRNA & AGTCGGCATCGTTTATGGTC & CGAAAGCATTTGCCAAGAAT & X00686 \\
\hline FAS & GGTCGTTTCTCCATTAAATTCTCAT & CTAGAAACTTTCCCAGAAATCTTCC & NM_007988 \\
\hline CPT-1 & CCAGGCTACAGTGGGACATT & GAACTTGCCCATGTCCTTGT & AF017175 \\
\hline
\end{tabular}




\section{Determination of hepatic lipid peroxidation and antioxidant enzymes}

Malondialdehyde (MDA) is a product of the lipid peroxidation and frequently used as a biomarker of oxidative stress [31]. In present study, the lipid peroxidation was determined by measuring the formation of thiobarbituric acid-reactive substances (TBARS) spectrophotometrically, and expressed as malondialdehyde (MDA) concentration [32]. The production of MDA, and activities of glutathione peroxidase (GPx), catalase (CAT), total superoxide dismutase (T-SOD), $\mathrm{Cu} / \mathrm{Zn}$-SOD, and Mn-SOD in liver tissues were measured with assay kits according to the instructions of manufacturer (Nanjing Jiancheng Bioengineering Institute, Nanjing, China).

\section{Statistical analysis}

Data were presented as means \pm standard error of mean (SEM). Differences between groups were analyzed by one-way analysis of variance (ANOVA) using SPSS 17.0 software, followed by Tukey's test. A value of $\mathrm{P}<0.05$ was considered significant.

\section{Results}

$\mathrm{H} 2 \mathrm{~S}$ decreases the liver weights of obese mice

After 16 weeks feeding, the mice were killed and the wet weight of the liver was monitored. Compared to the NFD fed mice, HFD fed mice showed increased liver weight (Figure $1 \mathrm{~A}, \mathrm{P}<0.01)$. H2S administration tended to significantly lower the liver weight compared to HFD group (Figure 1A, $\mathrm{P}<0.05$ ). HFD feeding did not affect the liver/body weight ratio (Figure $1 \mathrm{~B}$ ). H2S treatment markedly reduced the liver/body weight ratio (Figure 1B, $\mathrm{P}<0.05)$.

\section{$\mathrm{H} 2 \mathrm{~S}$ reduces the accumulation of lipids in the liver of} obese mice

Representative photomicrographs of liver $\mathrm{H} \& \mathrm{E}$ as well as ORO staining and quantitative analysis of hepatic triglyceride content were shown in Figure 2. Compared to NFD group, HFD caused significant vacuolar degeneration of hepatocytes, disruption of normal hepatic lobules, and inflammatory cells infiltration in H\&E-stained liver sections (Figure 2A). HFD-induced fat accumulation was characterized mainly by microvesicular steatosis as shown by ORO staining (Figure 2A), suggesting impaired mitochondrial function [33]. Compared to HFD group, H2S treatment significantly reduced hepatic lipid droplets and macrovesicular steatosis (Figure 2B, $\mathrm{C}, \mathrm{P}<0.01)$. Furthermore, the contents of TG and TC were analyzed to determine the extent of lipid accumulation in the liver. As shown in Figure 2D and 2E, HFD-fed mice had increased TG and TC contents in the liver compared to that of NFD group, while $\mathrm{H} 2 \mathrm{~S}$ treatment markedly reduced hepatic TG and TC contents $(\mathrm{P}<0.05)$. These results suggest that $\mathrm{H} 2 \mathrm{~S}$ may restore the liver from hepatic steatosis in obese mice.

\section{H2S modulates the expression of hepatic FAS and CPT-1}

FAS and CPT are two representative enzymes involved in lipid biogenesis and energy expenditure [33]. The expression levels of FAS and CPT-1 were assessed to investigate whether $\mathrm{H} 2 \mathrm{~S}$ would affect lipid metabolism in the liver. Although high-fat feeding markedly increased the mRNA expression level of FAS (Figure 3A, B, P < 0.05), there was no significant change in FAS protein expression between the NFD and HFD groups (Figure 3D). Compared to HFD group, H2S treatment reduced FAS expression at both the mRNA and protein levels (Figure 3A, B, D, $\mathrm{P}<0.05)$. The mRNA and protein expression levels of CPT-1 were significant lower in HFD group than that in NFD group (Figure 3A, C, E, P $<0.01$ ), while $\mathrm{H} 2 \mathrm{~S}$ treatment increased CPT-1 expression significantly at both the mRNA and protein levels (Figure 3A, C, E, $\mathrm{P}<0.05)$. Down-regulation of FAS in liver indicates that the synthesis of lipids decreased. Up-regulation of CPT-1 suggests that the expenditure of lipids increased.
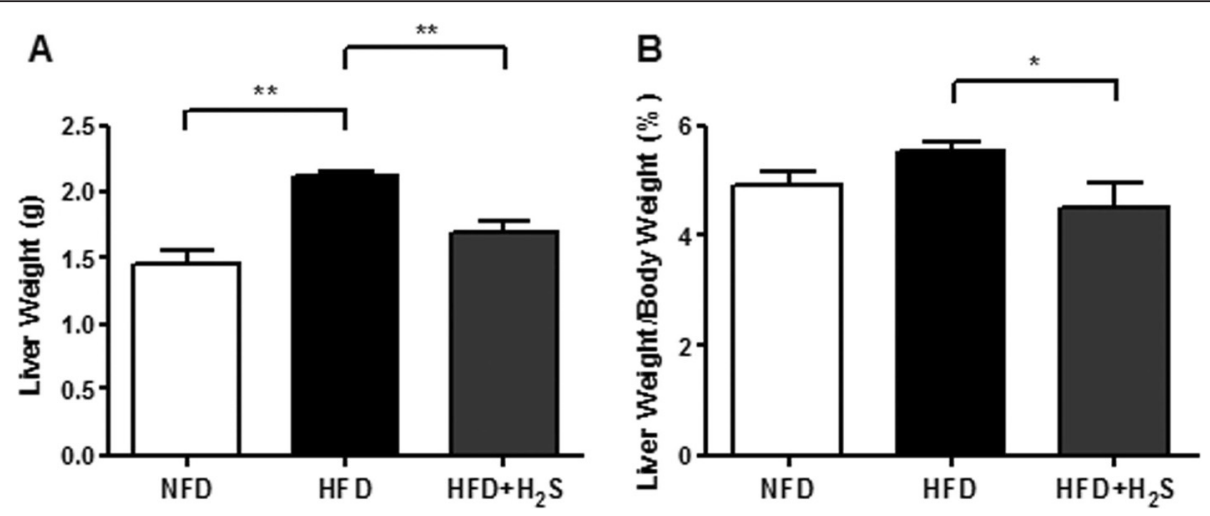

Figure 1 Effects of $\mathrm{H} 2 \mathrm{~S}$ on the liver mass of HFD-induced obese mice. (A) Liver weight. (B) The ratio of liver weight vs body weight. Data are means \pm SEM $(n=10) .{ }^{*} p<0.05,{ }^{* *} p<0.01$ vs. HFD group. 


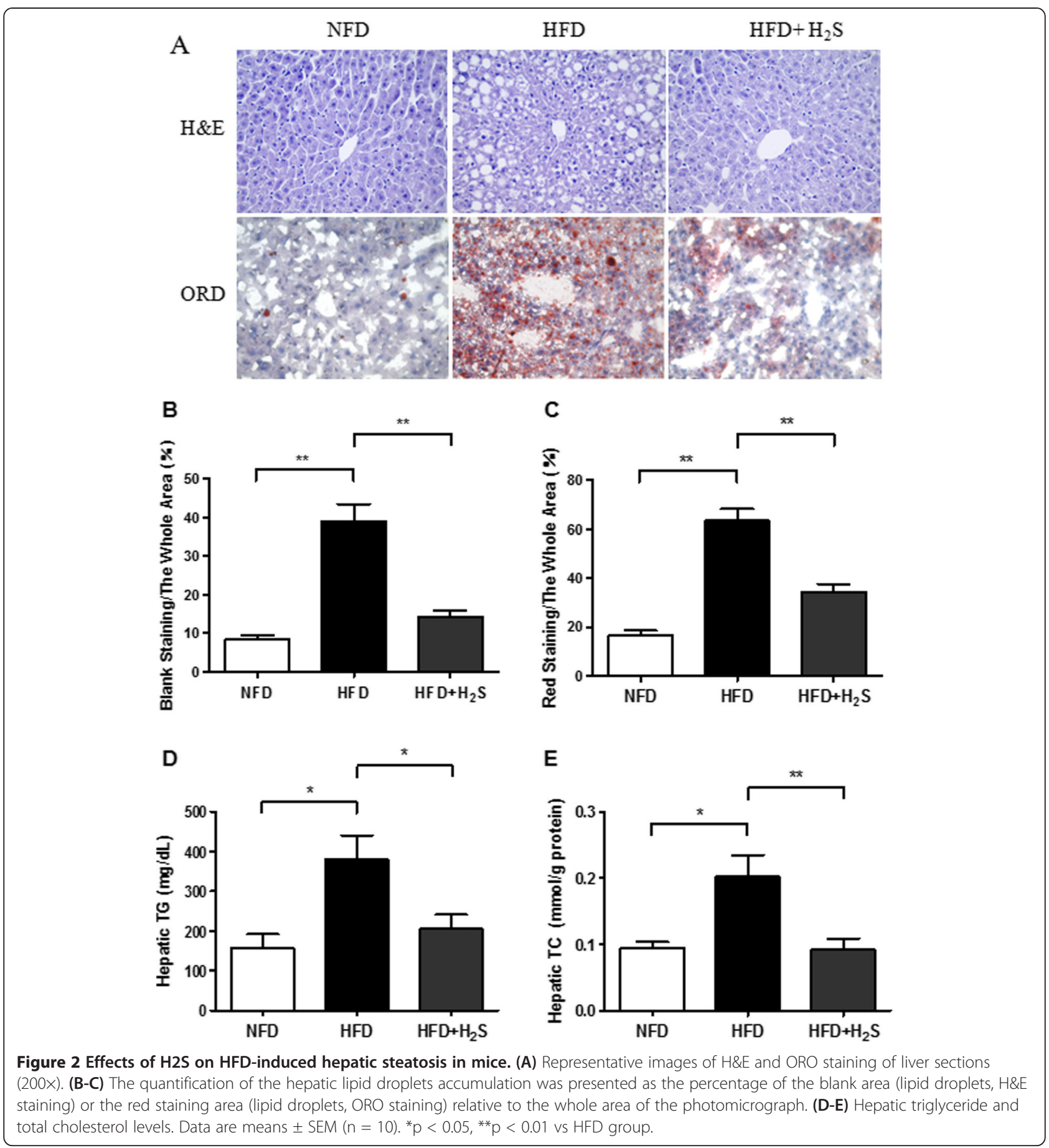

These results indicate that $\mathrm{H} 2 \mathrm{~S}$ reduces the accumulation of lipids in liver possibly through down-regulation of FAS and up-regulation of CPT-1.

\section{$\mathrm{H} 2 \mathrm{~S}$ regulates lipid peroxidation and antioxidant enzyme} activities in liver

HFD feeding significantly increased hepatic MDA formation (end-product of lipid peroxidation, as a biomarker of oxidative stress) and suppressed the activities of hepatic antioxidant enzymes including GPx, T-SOD, $\mathrm{Cu} / \mathrm{Zn}-\mathrm{SOD}$, and Mn-SOD. H2S treatment markedly decreased hepatic MDA formation and restored the activities of hepatic GPx, T-SOD, Cu/Zn-SOD, and Mn-SOD (Figure 4A, B, D, E, F, P < 0.05). No significant differences were observed in the catalytic activity of CAT among groups, suggesting that HFD feeding and H2S treatment did not affect the activity of CAT (Figure 4C). These results suggest that $\mathrm{H} 2 \mathrm{~S}$ protects the liver injury 

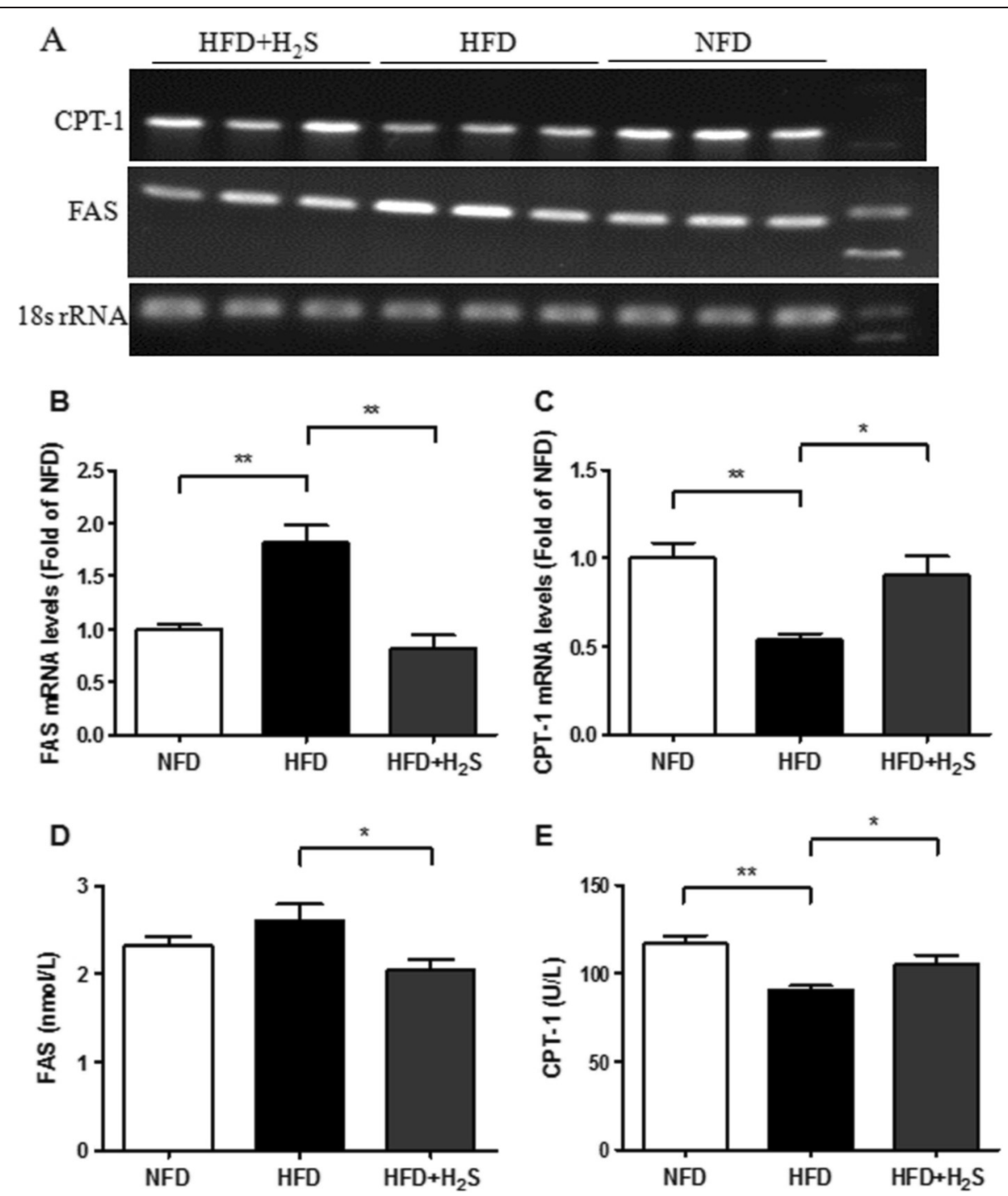

Figure 3 Effects of H2S on mRNA and protein levels of FAS and CPT-1 in the liver of HFD-fed mice. (A-C) The mRNA levels of FAS and CPT-1 were examined by RT-PCR $(n=3)$. (D-E) The protein levels of FAS and CPT-1 were examined by ELISA $(n=10)$. Data are means \pm SEM. ${ }^{*} p<0.05,{ }^{* *} p<0.01$ vs HFD group.

induced by HFD feeding through reducing the oxidative stress and increasing the activities of the antioxidant enzymes in the liver of HFD fed mice.

\section{Discussion}

The present study was designed to determine the effects of $\mathrm{H} 2 \mathrm{~S}$ on hepatic steatosis using a mouse model of HFD-induced obesity. We found that the administration of $\mathrm{H} 2 \mathrm{~S}$ donor NaHS could (1) reduce the liver weight; (2) reduce the contents of TG and TC in hepatocytes; (3) increase CPT-1 expression and decrease FAS expression; (4) increase the activities of antioxidant enzymes such as SOD and GPx. Based on our findings, we proposed a possible mechanism of $\mathrm{H} 2 \mathrm{~S}$ in mitigating fatty liver as shown in Figure 5. H2S can restore the liver from steatosis through suppressing hepatic fat accumulation by down-regulation of FAS and up-regulation of CPT-1. Additionally, H2S mitigates the fatty liver through up-regulating the activities of antioxidant enzymes (GPx and SOD). Take together, H2S products may be used as a therapeutical drug for the NAFLD.

Previous studies have demonstrated that a dramatic increase in overall liver weight and/or liver/body weight ratio could be observed in obese mice [34,35]. In the current study, HFD feeding resulted in a significant increase in overall liver weight, but did not change the liver/body weight ratio. However, H2S administration significantly reduced both the liver weight and liver/body weight ratio. Taken together, our results demonstrate that H2S has the ability to decrease liver weight in HFD-fed mice. 

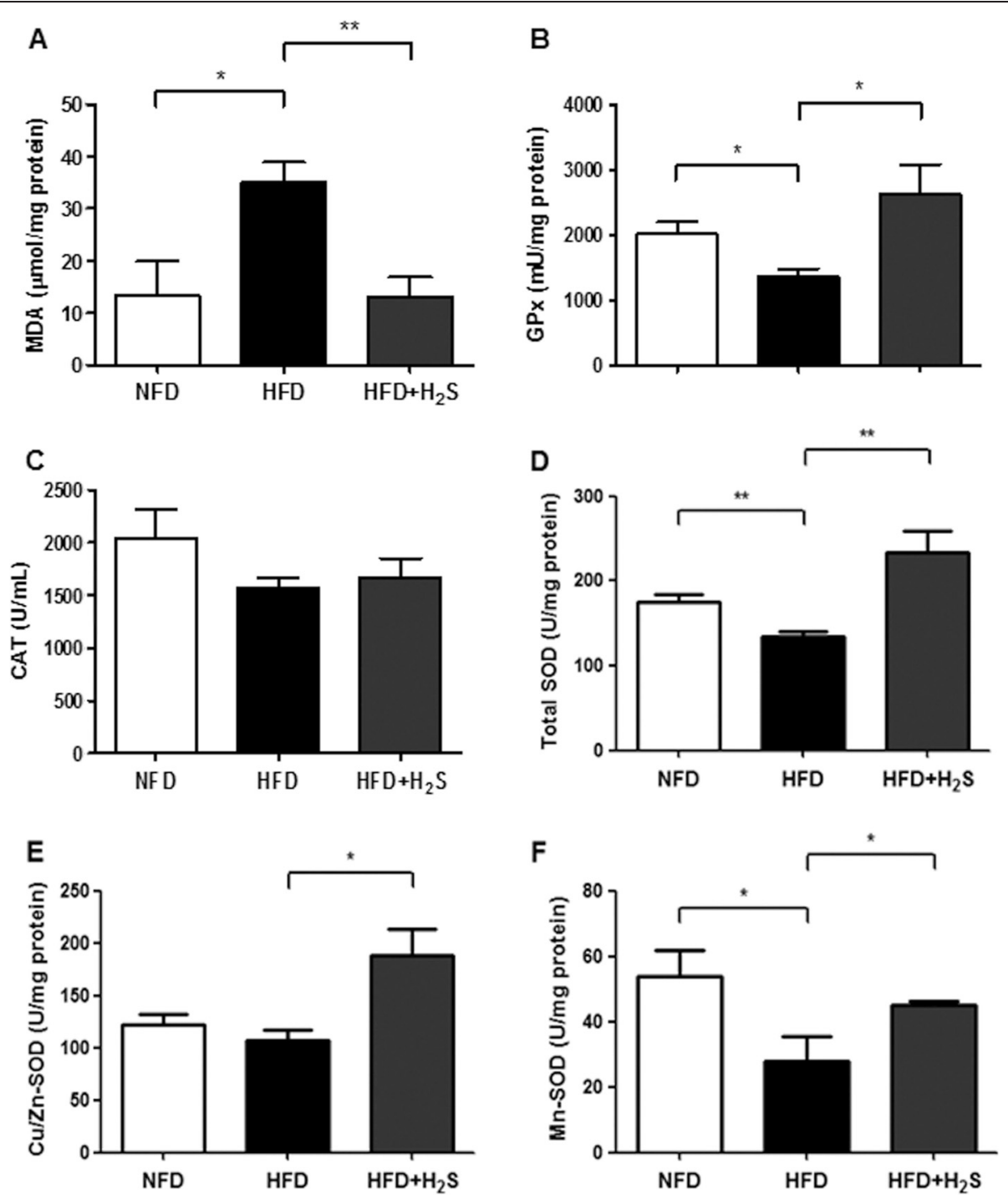

Figure 4 Effects of $\mathrm{H} 2 \mathrm{~S}$ on the MDA levels and the activities of antioxidant enzymes in the liver of HFD-fed mice. (A) MDA levels. (B-F) The activities of GPx, CAT, total SOD, Cu/Zn-SOD, and Mn-SOD. Data are means \pm SEM $(n=10) .{ }^{*} p<0.05$, ** $p<0.01$ vs HFD group.

As a central metabolic organ, the liver plays a vital role in various aspects of lipid and glucose metabolism in response to nutritional and hormonal signals [4,36]. Liver dysfunction could lead to dysregulation of lipid metabolism, including alterations in fatty acid biosynthesis, beta-oxidation and very low density lipoprotein (VLDL) secretion, which subsequently influences serum levels of cholesterol and lipoproteins $[4,37,38]$. In the present study, our observation proved obvious fat accumulation in the liver after feeding of mice with $45 \%$ HFD for 16 weeks, which was evidenced by H\&E and ORO staining (Figure 2). These phenotypic alterations were clearly abolished by $\mathrm{H} 2 \mathrm{~S}$ treatment for 4 weeks. In addition, H2S administration significantly reduced the hepatic triglyceride and cholesterol levels in HFD-induced obese mice, which was similar to a previous study that it inhibited cholesterol and triglyceride synthesis in liver of MCD diet-fed rats [9].

FAS and CPT-1 are two representative enzymes which play important roles in lipid biogenesis and energy expenditure [33]. FAS could effectively convert acetyl-CoA and malonyl-CoA into palmitate in a process that requires NADPH, which is supplied by both the pyruvate cycle and the pentose phosphate pathway [39]. CPT-1, an enzyme that resides in the outer mitochondrial membrane, is responsible for facilitating the entry of fatty acids into the mitochondria for oxidation [40,41]. In this study, H2S treatment markedly reduced FAS expression at both the mRNA and protein levels compared to the HFD group. In contrast, the increased expression of CPT-1 was observed in HFD + H2S group. These results suggest that $\mathrm{H} 2 \mathrm{~S}$ might prevent the development of fatty 


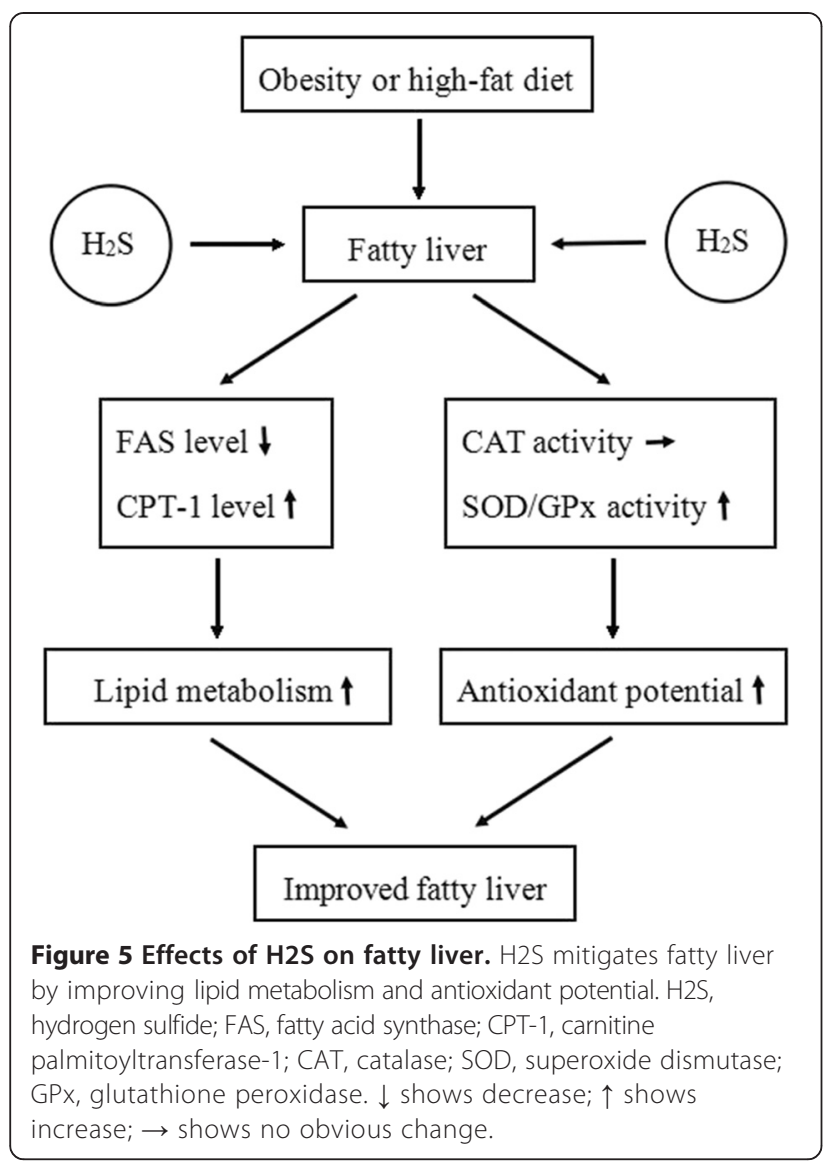

liver by modulating the expression of hepatic lipidregulating enzymes.

Reactive oxygen species (ROS) are free radicals derived from oxygen and non-radical species such as hydrogen peroxide (H2O2) [42]. Under normal physiological conditions, the levels of ROS generated from the oxidative processes within the cell can be effectively reduced by the antioxidant defense systems, which represent a number of enzymes such as SOD, CAT, and GPx, as well as non-enzymes include glutathione (GSH), vitamin E, vitamin A, and vitamin C $[43,44]$. Recent studies have indicated that ROS plays an important role in various liver diseases as a significant causative factor, including NAFLD/ NASH $[43,45,46]$. Oxidative stress is defined as an imbalance between the formation of ROS and the antioxidant defense capacity [47]. A central measurement of oxidative stress is lipid peroxidation (LPO), as indicated by MDA levels, which can contribute to a consequence of cellular damage [44]. It has been reported that H2S could prevent the elevation of MDA induced by MCD feeding [9]. Our results showed that $\mathrm{H} 2 \mathrm{~S}$ treatment significantly decreased hepatic MDA formation in HFD group. On the other hand, our present study found HFD diet declined hepatic antioxidative defense via down-regulating the activities of GPx and SOD. H2S effectively restored the activities of these enzymes. These findings indicate that $\mathrm{H} 2 \mathrm{~S}$ could provide anti-oxidative effects against HFD-induced hepatotoxicity.

Based on the results obtained in the present study, it is concluded that administration of $\mathrm{H} 2 \mathrm{~S}$ could mitigate the fatty liver by suppressing hepatic fat accumulation through down-regulation of FAS and up-regulation of CPT-1 in HFD-fed obese mice. In addition, H2S may suppress oxidative stress by increasing the activities of antioxidant enzymes such as GPx and SOD. These results suggest that $\mathrm{H} 2 \mathrm{~S}$ can play an important role in regulating the lipid and antioxidant metabolism. However, further studies are needed to elucidate the precise underlying mechanism of lipid metabolism and antioxidant activities of $\mathrm{H} 2 \mathrm{~S}$.

\section{Conclusion}

In conclusion, we proved that $\mathrm{H} 2 \mathrm{~S}$ can restore the liver from steatosis in obese mice. As shown in Figure 5, H2S mitigates the fatty liver most likely through (1) suppressing hepatic fat accumulation by down-regulation of FAS and up-regulation of CPT-1; (2) up-regulating the activities of antioxidant enzymes (GPx and SOD).

\section{Abbreviations}

NAFLD: Nonalcoholic fatty liver disease; HFD: High fat diet; NFD: Normal fat diet; TG: Triglyceride; TC: Total cholesterol; FAS: Fatty acid synthase; CPT-1: Carnitine palmitoyltransferase-1; MDA: Malondialdehyde; SOD: Superoxide dismutase; GPx: Glutathione peroxidase (GPx).

\section{Competing interests}

The authors declare that they have no competing interests.

\section{Authors' contributions}

DW participated in the design of the study and the preparation of the manuscript. NZ, KQ, HC, ZS, BG, and YZ carried out all the animal experiments. $W P, C H F$, and SJ participated in the experimental design and data processing. $M Z, A J$, and $Y L$ participated in the overall design of the study and manuscript writing. All authors read and approved the final manuscript.

\section{Acknowledgements}

This work was supported by the grant 132300410012 (YL) from Henan Provincial Science \& Technology, China and National Natural Science Foundation of China Grant 81471174 (MX).

\section{Author details}

${ }^{1}$ Medical College of Henan University, Kaifeng 475004, Henan, China. ${ }^{2}$ Institute of Environmental Medicine of Henan University, Kaifeng 475004, China. ${ }^{3}$ Nursing College of Henan University, Kaifeng 475004, China. ${ }^{4}$ Department of Neurology, the First Affiliated Hospital, Institute of Neurological Disorder, Henan University, Kaifeng 475004, China. ${ }^{5}$ Huaihe Hospital of Henan University, Kaifeng 475004, China.

Received: 26 November 2014 Accepted: 16 December 2014 Published online: 10 January 2015

\section{References}

1. Cohen JC, Horton JD, Hobbs HH. Human fatty liver disease: old questions and new insights. Science. 2011;332:1519-23.

2. Birkenfeld AL, Shulman GI. Nonalcoholic fatty liver disease, hepatic insulin resistance, and type 2 diabetes. Hepatology. 2014;59:713-23.

3. Kimura $\mathrm{H}$. The physiological role of hydrogen sulfide and beyond. Nitric Oxide. 2014;41:4-10.

4. Mani S, Cao W, Wu L, Wang R. Hydrogen sulfide and the liver. Nitric Oxide. 2014;41C:62-71. 
5. Xue R, Hao DD, Sun JP, Li WW, Zhao MM, Li XH, et al. Hydrogen sulfide treatment promotes glucose uptake by increasing insulin receptor sensitivity and ameliorates kidney lesions in type 2 diabetes. Antioxid Redox Signal. 2013;19:5-23.

6. Yang G, Tang G, Zhang L, Wu L, Wang R. The pathogenic role of cystathionine gamma-lyase/hydrogen sulfide in streptozotocin-induced diabetes in mice. Am J Pathol. 2011;179:869-79.

7. Tan G, Pan S, Li J, Dong X, Kang K, Zhao M, et al. Hydrogen sulfide attenuates carbon tetrachloride-induced hepatotoxicity, liver cirrhosis and portal hypertension in rats. PLoS One. 2011;6:e25943.

8. Jain SK, Manna P, Micinski D, Lieblong BJ, Kahlon G, Morehead L, et al. In African American type 2 diabetic patients, is vitamin $D$ deficiency associated with lower blood levels of hydrogen sulfide and cyclic adenosine monophosphate, and elevated oxidative stress? Antioxid Redox Signal. 2013;18:1154-8.

9. Luo ZL, Tang LJ, Wang T, Dai RW, Ren JD, Cheng L, et al. Effects of treatment with hydrogen sulfide on methionine-choline deficient diet-induced non-alcoholic steatohepatitis in rats. J Gastroenterol Hepatol. 2014;29:215-22.

10. Kang K, Zhao M, Jiang H, Tan G, Pan S, Sun X. Role of hydrogen sulfide in hepatic ischemia-reperfusion-induced injury in rats. Liver Transpl. 2009;15:1306-14.

11. Morsy MA, Ibrahim SA, Abdelwahab SA, Zedan MZ, Elbitar HI. Curative effects of hydrogen sulfide against acetaminophen-induced hepatotoxicity in mice. Life Sci. 2010;87:692-8.

12. Helmy N, Prip-Buus C, Vons C, Lenoir V, Abou-Hamdan A, Guedouari-Bounihi H, et al. Oxidation of hydrogen sulfide by human liver mitochondria. Nitric Oxide. 2014;41:105-12.

13. Wang CN, Liu YJ, Duan GL, Zhao W, Li XH, Zhu XY, et al. CBS and CSE Are Critical for Maintenance of Mitochondrial Function and Glucocorticoid Production in Adrenal Cortex. Antioxid Redox Signal. 2014;21:2192-207.

14. Bos EM, Wang R, Snijder PM, Boersema M, Damman J, Fu M, et al. Cystathionine gamma-lyase protects against renal ischemia/reperfusion by modulating oxidative stress. J Am Soc Nephrol. 2013;24:759-70.

15. Du JT, Li W, Yang JY, Tang CS, Li Q, Jin HF. Hydrogen sulfide is endogenously generated in rat skeletal muscle and exerts a protective effect against oxidative stress. Chin Med J (Engl). 2013;126:930-6.

16. Yao LL, Huang XW, Wang YG, Cao YX, Zhang CC, Zhu YC. Hydrogen sulfide protects cardiomyocytes from hypoxia/reoxygenation-induced apoptosis by preventing GSK-3beta-dependent opening of mPTP. Am J Physiol Heart Circ Physiol. 2010;298:H1310-9.

17. Lo Faro ML, Fox B, Whatmore JL, Winyard PG, Whiteman M. Hydrogen sulfide and nitric oxide interactions in inflammation. Nitric Oxide. 2014:41:38-47.

18. Szabo C, Coletta C, Chao C, Modis K, Szczesny B, Papapetropoulos A, et al. Tumor-derived hydrogen sulfide, produced by cystathionine-beta-synthase, stimulates bioenergetics, cell proliferation, and angiogenesis in colon cancer. Proc Natl Acad Sci U S A. 2013;110:12474-9.

19. Coletta C, Papapetropoulos A, Erdelyi K, Olah G, Modis K, Panopoulos P, et al. Hydrogen sulfide and nitric oxide are mutually dependent in the regulation of angiogenesis and endothelium-dependent vasorelaxation. Proc Natl Acad Sci U S A. 2012;109:9161-6.

20. Wang MJ, Cai WJ, Li N, Ding YJ, Chen Y, Zhu YC. The hydrogen sulfide donor NaHS promotes angiogenesis in a rat model of hind limb ischemia. Antioxid Redox Signal. 2010;12:1065-77.

21. Polhemus DJ, DJ L. Emergence of Hydrogen Sulfide as an Endogenous Gaseous Signaling Molecule in Cardiovascular Disease. Circ Res. 2014;114:730-7.

22. Ge SN, Zhao MM, Wu DD, Chen Y, Wang Y, Zhu JH, et al. Hydrogen Sulfide Targets EGFR Cys797/Cys798 Residues to Induce Na/K-ATPase Endocytosis and Inhibition in Renal Tubular Epithelial Cells and Increase Sodium Excretion in Chronic Salt-Loaded Rats. Antioxid Redox Signal. 2014;21:2061-82.

23. Yang G, Wu L, Jiang B, Yang W, Qi J, Cao K, et al. H2S as a physiologic vasorelaxant: hypertension in mice with deletion of cystathionine gamma-lyase. Science. 2008;322:587-90.

24. Shirozu K, Tokuda K, Marutani E, Lefer D, Wang R, Ichinose F. Cystathionine gamma-lyase deficiency protects mice from galactosamine/lipopolysaccharideinduced acute liver failure. Antioxid Redox Signal. 2014;20:204-16.

25. Peh MT, Anwar AB, Ng DS, Atan MS, Kumar SD, Moore PK. Effect of feeding a high fat diet on hydrogen sulfide (HS) metabolism in the mouse. Nitric Oxide. 2014;41:138-45

26. Hwang SY, Sarna LK, Siow YL, Karmin O. High-fat diet stimulates hepatic cystathionine beta-synthase and cystathionine gamma-lyase expression. Can J Physiol Pharmacol. 2013;91:913-9.
27. Gao M, Bu L, Ma Y, Liu D. Concurrent activation of liver $X$ receptor and peroxisome proliferator-activated receptor alpha exacerbates hepatic steatosis in high fat diet-induced obese mice. PLoS One. 2013;8:e65641.

28. Park MY, Jang HH, Kim JB, Yoon HN, Lee JY, Lee YM, et al. Hog millet (Panicum miliaceum L.)-supplemented diet ameliorates hyperlipidemia and hepatic lipid accumulation in C57BL/6 J-ob/ob mice. Nutr Res Pract. 2011;5:511-9.

29. Ko C, O'Rourke SM, Huang LS. A fish oil diet produces different degrees of suppression of apoB and triglyceride secretion in human apoB transgenic mouse strains. J Lipid Res. 2003:44:1946-55.

30. Li Y, Tong X, Rumala C, Clemons K, Wang S. Thrombospondin1 deficiency reduces obesity-associated inflammation and improves insulin sensitivity in a diet-induced obese mouse model. PLoS One. 2011;6:e26656.

31. Ayala A, Munoz MF, Arguelles S. Lipid peroxidation: production, metabolism, and signaling mechanisms of malondialdehyde and 4-hydroxy-2-nonenal. Oxid Med Cell Longev. 2014;2014:360438.

32. Wang $T$, Yang $P$, Zhan $Y, X i a ~ L$, Hua Z, Zhang J. Deletion of circadian gene Per1 alleviates acute ethanol-induced hepatotoxicity in mice. Toxicology. 2013;314:193-201.

33. Song M, Schuschke DA, Zhou Z, Chen T, Pierce Jr WM, Wang R, et al. High fructose feeding induces copper deficiency in Sprague-Dawley rats: a novel mechanism for obesity related fatty liver. J Hepatol. 2012;56:433-40.

34. Jwa H, Choi Y, Park UH, Um SJ, Yoon SK, Park T. Piperine, an LXRalpha antagonist, protects against hepatic steatosis and improves insulin signaling in mice fed a high-fat diet. Biochem Pharmacol. 2012;84:1501-10.

35. DeAngelis RA, Markiewski MM, Taub R, Lambris JD. A high-fat diet impairs liver regeneration in C57BL/6 mice through overexpression of the NF-kappaB inhibitor, IkappaBalpha. Hepatology. 2005:42:1148-57.

36. Schug TT, Li X. Sirtuin 1 in lipid metabolism and obesity. Ann Med. 2011:43:198-211.

37. Donnelly KL, Smith Cl, Schwarzenberg SJ, Jessurun J, Boldt MD, Parks EJ. Sources of fatty acids stored in liver and secreted via lipoproteins in patients with nonalcoholic fatty liver disease. J Clin Invest. 2005;115:1343-51.

38. Savage DB, Semple RK. Recent insights into fatty liver, metabolic dyslipidaemia and their links to insulin resistance. Curr Opin Lipidol. 2010;21:329-36.

39. Peinado JR, Quiros PM, Pulido MR, Marino G, Martinez-Chantar ML, Vazquez-Martinez R, et al. Proteomic profiling of adipose tissue from Zmpste24-/- mice, a model of lipodystrophy and premature aging, reveals major changes in mitochondrial function and vimentin processing. Mol Cell Proteomics. 2011;10(M111):008094.

40. Brown NF, Hill JK, Esser V, Kirkland JL, Corkey BE, Foster DW, et al. Mouse white adipocytes and 3 T3-L1 cells display an anomalous pattern of carnitine palmitoyltransferase (CPT) I isoform expression during differentiation. Inter-tissue and inter-species expression of CPT I and CPT ॥ enzymes. Biochem J. 1997;327(Pt 1):225-31.

41. McGarry JD, Brown NF. The mitochondrial carnitine palmitoyltransferase system. From concept to molecular analysis. Eur J Biochem. 1997;244:1-14.

42. Gill SS, Tuteja N. Reactive oxygen species and antioxidant machinery in abiotic stress tolerance in crop plants. Plant Physiol Biochem. 2010;48:909-30.

43. Leung TM, Nieto N. CYP2E1 and oxidant stress in alcoholic and non-alcoholic fatty liver disease. J Hepatol. 2013;58:395-8.

44. Li Y, Li M, Shi J, Yang X, Wang Z. Hepatic antioxidative responses to PCDPSs and estimated short-term biotoxicity in freshwater fish. Aquat Toxicol. 2012;120-121:90-8.

45. Leung C, Herath CB, Jia Z, Goodwin M, Mak KY, Watt MJ, et al. Dietary glycotoxins exacerbate progression of experimental fatty liver disease. J Hepatol. 2014;60:832-8.

46. van de Wier B, Balk JM, Haenen GR, Giamouridis D, Bakker JA, Bast BC, et al Elevated citrate levels in non-alcoholic fatty liver disease: the potential of citrate to promote radical production. FEBS Lett. 2013;587:2461-6.

47. Besse-Patin A, Estall JL. An Intimate Relationship between ROS and Insulin Signalling: Implications for Antioxidant Treatment of Fatty Liver Disease. Int J Cell Biol. 2014:2014:519153. 